A METHOD OF MEASURING THE POLARIZATION

OF HIGH MOAENTIM PROTON BEAMS

David G. Underwood

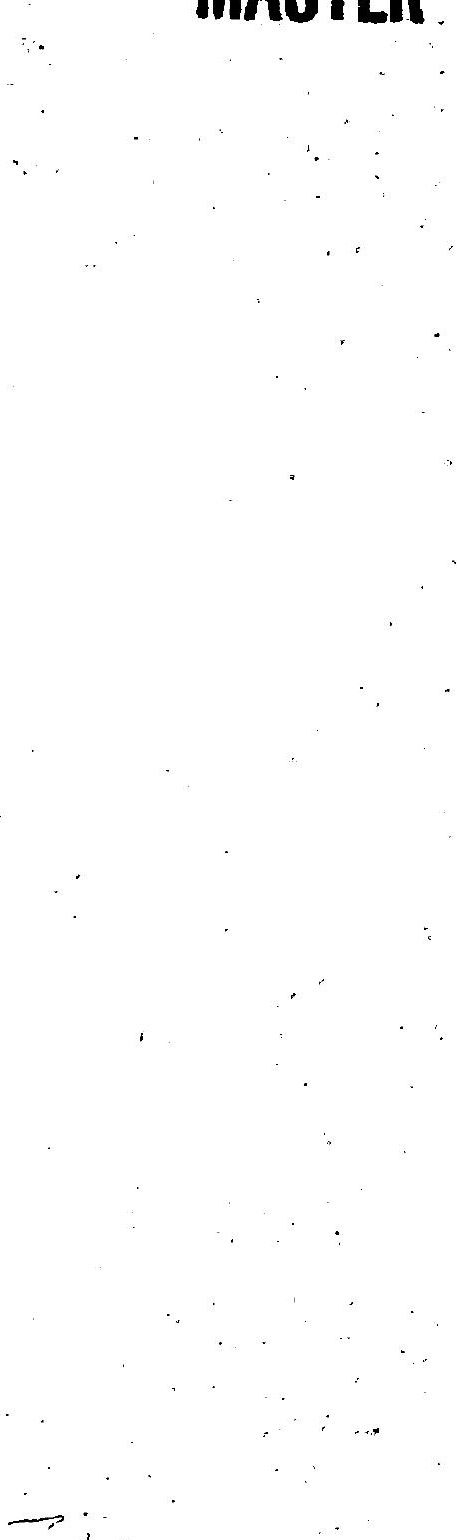


The facilities of Argonne National Laboratory are owned by the: United States Government. Under the terms of a contract (W-31-109-Eng-38) between the U. S. Energy Research and Development Administration, Argonine Universities Association and The University of Chicago, the University employs the staff and operates the Laboratory in accordance with policies and programs formulated, approved and reviewed by the Association: The University of Arizona MEMBERS Carnegie-Mellon University Case Western Reserve University The University of Chicago University of Cincinnati. Illinois:Institute of Technology University of Illinois Indiana University Iowa State University; The University of Iowa

\section{$\therefore \therefore$}

\section{ARGONNE UNIVERSITIES ASSOCIATION}

Kansas State University

The University of Kansas Layola University Marquette University Michigan State University The Univer sity of Michigan University of Minnesota University of Missouri Northwestern, University University of Nótre Dame

\section{The Ohio State University} Ohio University -The Pennsylvania State University. Puxdue Univer sity Saint . Lauis University Southern Illinois University The University of Texas at Austin Washington University Wayne State University The University of Wisconsin

\section{NOTICE}

This report was prepared as an account of work sponsored by the United States Government. Neither the United States nor the United States Energy Research and Development Administration, nor any of their employees, nor any of their contractor"s, subcontractors, or their employees, makes any warranty, express or implied, or assumes any. legal liabilityor responsibility for the accuracy, completeness or usefulness of any information, apparatus, product or process disclosed, or represents that its use would not infringe privately-owned rights: Mention of commercial products, their manufacturers, or their suppliers in this publication does not imply or connote approval or disapproval of the product by Argonne National Laboratory or the U. S. Energy Research and-Development Administration. 


\title{
A METHOD OF MEASURING THE POLARIZATION
}

\section{OF HIGH MONENTUM, PROTON BEAMS}

\author{
David. G. Underwood
}

Argonne National Laboratory

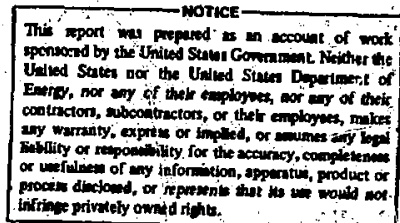

\section{ABSTRACT}

A method of measuring the polarization of high

momentum proton beams is proposed. This method utilizes the Primakoff effect and relates asymetries at.high eriergy to large asymmetries already measured at low energy. Such a new method is essential for the success of future ex- .. periments at energies where present methods are no longer feasable.

By acceptance of this article; the publisher or recipient acknowledges the U.S. Government's right to. retain a nonexclusive, royalty-free license in and to any copyright: covering the article. 


\section{INTRODUCTION}

Experiments are now being proposed ${ }^{1,2}$ which will use high momentum $(<300 \mathrm{GeV} / \mathrm{c})$ polarized proton beams derived from lambda decay. For these experiments it is essential to know the polarization of the beam accurately and the proposed methods of calculating polarization are probably not adequate due to backgrounds and uncertainties in geometry. Also, experiments now in progress ${ }^{3}$ are somewhat dependent on knowing the polarization in ordinary high momentum beams $(.2<\mathrm{x}<.8)$. We discuss some methods of measuring beain polarization at high energy and describe one particularly attractive method in detail.

\section{DISCUSSION}

Some methods used to measure beam polarization at lower momentum $(\$ 12 \mathrm{GeV} / \mathrm{c}$ ) are known not to work well at higher momentum, e.g., elastic scattering from carbon or hydrogen. The effects are very small at low $\left.t\left(\omega-2(\mathrm{GeV}){ }^{2}\right)^{2}\right)$ and the cross sections are too small at higher $t\left(v-1,4(\mathrm{GeV} / \mathrm{c})^{2}\right)$ as measured in Fermilab Exp. \#61. 3

One proposed method involves looking at $\Lambda^{0}$ decay for $\Lambda^{\circ}$ produced in the forward direction from protons. In the exact forward direction the $A^{\circ}$ must carry the spin direction of the proton due to angular momentum conservation.

This method may not be applicable if flip amplitudes exist 
at smaller values of than can be resolved experimentally. In diffractive production of $n *$ at $|t|$ of about $.02 \mathrm{GeV} / \mathrm{c}^{2}$, angular distributions implying the existence of large helicity amplitudes and some flip amplitudes have been observed. ${ }^{5}$ While these amplitudes may not be fundamental in the diffractive interaction; they do exist in the observable final state. Such amplitudes could be present for lambdas produced diffractively or multi-peripherally.

There are a number of low momentum transfer high energy processes which are related to low energy processes by crossing or time reversal. For exampie, the deck effect diffractive process $\mathrm{PA} \rightarrow \mathrm{A} \mathrm{P}^{+} \pi^{-}$(Fig. 1) looks like $500 \mathrm{MeV} / \mathrm{c}^{\prime} \pi$ elastic scattering with the complication that the pion is off the mass shell part of the time and there may be final state interactions.

In principle one could relate the asymmetry parameter in $\pi \mathrm{p}_{\uparrow}$ scattering measured at various energies and angles to asymetries expected in the $\mathrm{D} \pi^{+} \pi^{-}$system for polarized incident $300 \mathrm{GeV} / \mathrm{c}$ protons. Such a calculation may be possible in limited kinematic regions, but one would probably want to calibrate by using protons of known polarization from $\Lambda^{\circ}$ decay in a spectrometer such as that in Fermilab Experiment \#8, 4 They have already proposed to do diffraction dissociation of neutrons in their $\Lambda^{\circ}$ spèctrometer as a measurement of backgrounds for a proposed $\mathrm{K}$ beam.

There is, however, a much more appealing channel for which calculations can be done, for which there are essentially no final state interactions not already present in the 10w energy data, and for which time reversal symmetry (and the "calculations) have been experimentally verified to about $\pm 3 \%$. 
The processes of radiative decay of meson and baryon resonances, production of such resonances in the coulomb field of nuclei, and photoproduction of resonances can be related as discussed by Primakoff, 6 Good and Walker, 7 and many others. More specifically for our purposes one can calculate $\mathrm{P}+\mathrm{A}+\mathrm{A}+\mathrm{p} \pi^{\circ}$ in the coulomb production region from measurements of op $+\pi^{\circ}$ p at low energy. Such calculations apply to the general process and not just to resonance production.

For $\mathrm{P}^{\circ}$ masses above the $\Delta$ mass the total cross section, differential cross section, and many spin combinations have been measured ${ }^{8}$ or will soon be measured ${ }^{9}$ for $\gamma P \rightarrow \ddot{P}^{\circ}$. Typical results are shown in Fig. 2 :

The $\mathrm{P}_{\pi}^{\circ}$ final state was chosen because very precise measurements of both position and momentum of final state particles are needed. It is difficult to measure the neutron momentum from $\mathrm{n}^{+}$with a res lution of $1 \%$ of the beam momentum. The $\mathrm{P} \pi^{+}+\pi^{-}$ final state was not considered because photoproduction data in appropriate spin states are not yet adequate.

To measure the polarization of the proton beam, one would look at the angluar distributions in the Gottfied-Jackson or $t-$ helicity frame for appropriate $\mathrm{p}^{0}$ masses. These can probably be translated into a combination of hits of MWPC wires before and after an analysis magnet in conjunction with scintillator hits in a lead-scintillator $\pi$ detector Polarization could then be measured in real time with the on-iine computer for an experiment.

The system could be self-calibrated by carefully analyzing recorded events and doing background subtractions. The analyzIng power for a clean sample of events would be known from the 
photoproduction data.

Since the virtual photon direction is nearly antiparallel to the beam direction in the proton rest frame, and $t$ helicity frame, this system will analyze any polarization comr onent perpendicular to the beam. The beam spin can be rotated using the anomolous moment in order to measure any. polarization component.

An argument ${ }^{10}$ based on electromagnetic current conservation shows that only helicity \pm 1 states of the virtual protons in the coulomb field contribute to coulomb dissociation. Since there is momentum transfer between the nucleus and the proton but almost no energy transfer to the nucleus, the proton is off the mass shell or has invariant mass squared of $t$ (negative). At $2 \times t_{\min }$ where most of he production occurs this is $210^{-5}(\mathrm{GeV})^{2}$.

\section{SOME PRACTICAL CONSIDERATIONS}

The direct measurement of coulomb production at Fermi-Lab energies requires that the transverse momentum error on in- : dividual track measurements be less than $20 \mathrm{MeV} / \mathrm{c}$ and preferably less than $10 \mathrm{MeV} / \mathrm{c}$. The beam direction must then be measured to about $.03 \mathrm{mR}$ or $.3 \mathrm{~mm}$ in $10 \mathrm{~m}$. This resolution has been achieved in several experiments such as diffraction dissociation experiments 27 and 305, and measurements of coulomb interference in elastic scattering. Given this resolution, the angles of the pion or proton in the $t$ helicity frame (which corresponds to the S helicity frame in photo production) are known to about $\pm 5^{\circ}$. This resolution is adequate for cutting the data for comparison 
with photoproduction data or in order to use an angular region with high analyzing power.

It also becomes simple to measure polarization as a function of position or angle within the beam. The capability could be quite useful with low momentum high devergence beams where polarization is expected to vary with position or angle because of the effects of quadrupoles.

Differential cross sections and some polarization asymmetry data exist for $\gamma \mathrm{n} \rightarrow \pi^{-} \mathrm{p}$ at low energy $(500 \mathrm{MeV}$ to $1 . \mathrm{GeV})$ and total crioss sections and angular distributions exist for $n+P_{b}+P_{b}+p \pi^{-}$at $300 \mathrm{GeV}$ for $p \pi^{-}$masses from threshold up. This process will not have the same polarization effects as $\gamma \mathrm{p} \rightarrow \mathrm{p} \pi^{\circ}$ because of the charged current coupling of $\pi^{-}$but the effective mass distribution, $t$ distribution, and cross section in the coulomb dissociation process can serve to illustrate what would have to be measured and to what accuracy as shown in Fig. 3 .

The $\mathrm{p} \pi^{\circ}$ mass spectrum produced by coulomb dissociation dies out quickly above the $\Delta$ mass for two reasons: 1) the cross sections are small even in photoproduction. 2) there is a kinematic factor $\left(t_{\text {min }}\right)$ in coulomb production which suppresses higher masses for these reasons we would like to work near the $\Delta$ mass. At the central mass of the $\Delta$ the ratio of $\Delta$ production to non-resonant production may be too large to produce significant polarization effects by interference. On the low mass side of the $\Delta$ it may be experimentally difficult to obtain the required resolution ant acceptance: This leaves the high mass side of the $\Delta$. 
The proton asymmetry data $T(\theta)$ for $\pi^{\circ}$ photoproduction with a polarized target are onily available at present for masses above $1480 \mathrm{GeV}$. Asymmetries calculated from the 1969 walker amplitude fits ${ }^{11}$ (Fig.2) agree qualitatively, but not well enough to be used for decailed polarization measurements using lower mass $P \pi^{\mathrm{O}}$ systems.

A Japanese collaboration, reporting on $\pi^{+} n$ photoproduction at the 1976 conference on high energy physics with polarized beams and targets, ${ }^{9}$ expects to publish new data on $\mathrm{P \pi}^{\circ}$ photoproduction and a comparison with new amplitude analyses soon. The cross section for $p \pi^{0}$ coulomb production on $P_{b}$ is about $10 . \mathrm{mb}$ at $25 \mathrm{GeV} / \mathrm{c}$ and about $20 . \mathrm{mB}$ at $250 \mathrm{GeV} / \mathrm{c}$ for the relevant $t^{\prime}$ range $(|t|<.005)$. The total cross section for protons on $P_{b}$ is about $3000 \mathrm{mb}$. The target must be thin (< $\frac{1}{2}$ rad length) to obtain adequate directional resolution. Half a radiation length is about $3 \%$ of an interaction length. Thus, $2 \times 10^{-4}$ of the beam gives coulomb events, and perhaps $5 \%$ of this, or $1 \times 10^{-5}$ of the beam is useful for polarization analysis. At $1 \times 10^{7}$ beam per spill we could get a $3 \%$ polarization measurement in 10 spills. Since the polarization is controlled primarily by geometry and beamline settings, this seems like a reasonable time period ( $\sim 2$ minutes at Fermilab):

1. Letter of Intent on the study of spin effects at SPS energies by making use of a poiarized beam facility, CERN/SPSC/76-95, SPSC/I 87, Il November 1976.

2. Argonne Study Group on Fixed Target Hadron Interactions.

3. Elastic Scattering with a Polarized Target, Fermilab Exp 61. 
4. Experiments with neutral hyperons, Fermi-Lab Exp. 8.

5. Decay properties of $\mathrm{P}^{-}$systems produced in neutron dissociation at 50-300 GeV/C, J. Biel, E. J. Bleser, D. Duke, T. Ferbe1, D. Freytag, B, Gobbi, I. Kenah, J. Rosen, R. Ruchti, P. Slattery, and D. Underwood, Phys. Rev. Lett 36, 507 (1976).

6. H. Primakoff, Phys. Rev. 81; 899 (1951)

7. Coulomb Dissociation of Beam Particles, M. L. Good and W. D. Walker, Phys. Rev. 120, 1855 (1960).

8. International Symposium on Electron and Photon Interactions at High Energies, 1973, edited by H. Rollnik and W. Pfeil, North-Holland/American Elsevier.

9. Angular distributions of the polarized target asymetry on $\gamma P>\pi^{+} n$ at the energies of $0.5-0.8 \mathrm{GeV} ; \mathrm{M}$. Fukushima et al. high energy physics wit' polarized beams and targets, Argonne, 1976, A.I.P. Conference Proceedings No. 35, Particles and Fields Subseries No. 12.

10. C. Andersen, A. Halpern, H. Primakoff. Phys. Rev. Lett. 9 $512(1962)$.

11. Plienomenological Analysis of Single-Pion Photoproduction, R. L. Walker Phys. Rev. 182,1729 (1969). 


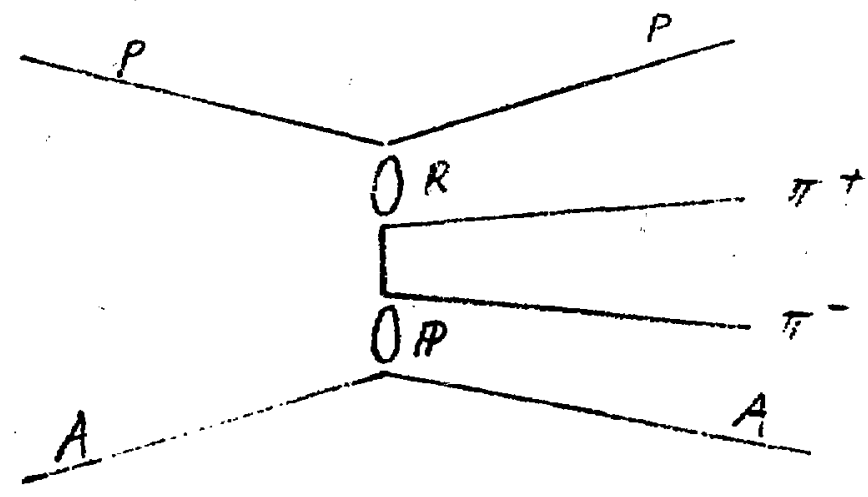

\section{FIGURE 1:}

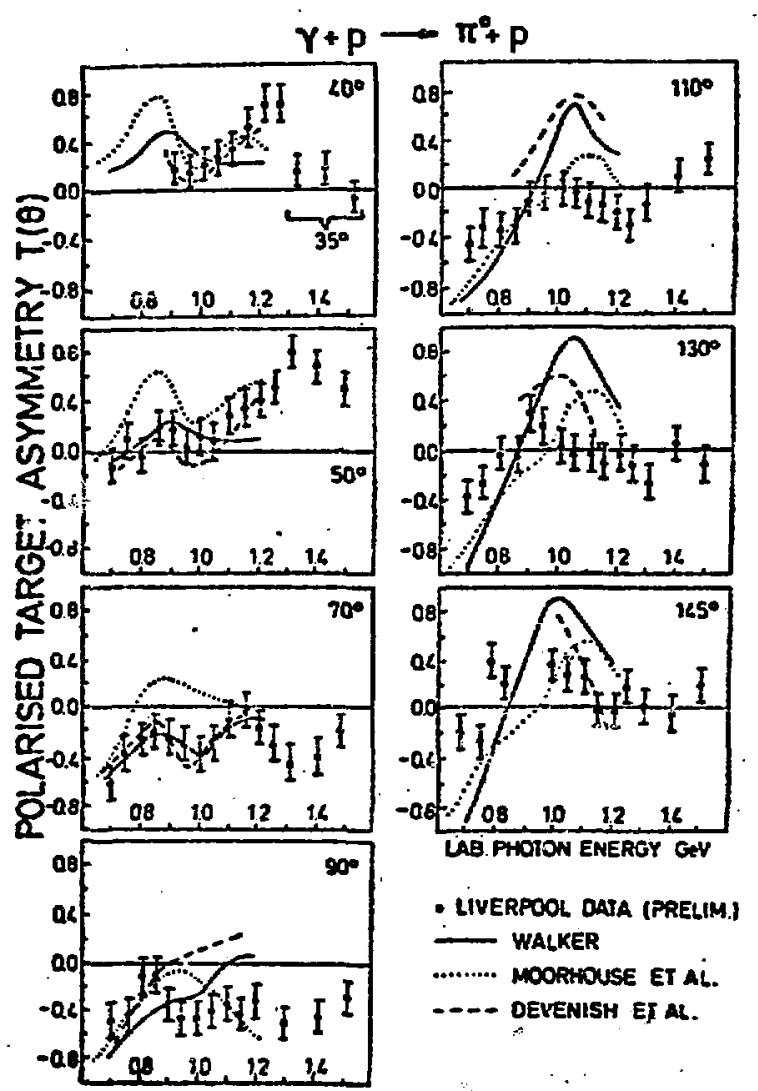

IIGURE 2: . Energy dependince of the poiarized target asymmetry. $T(t)$ for pion $\mathrm{cm}$ angles of $40^{\circ}, 50^{\circ}, 70^{\circ}, 110^{\circ}, 130^{\circ}$ and $145^{\circ}$. The curves show fits by Wialker MiO.R. and Devenüsh, Lith and Rankin 


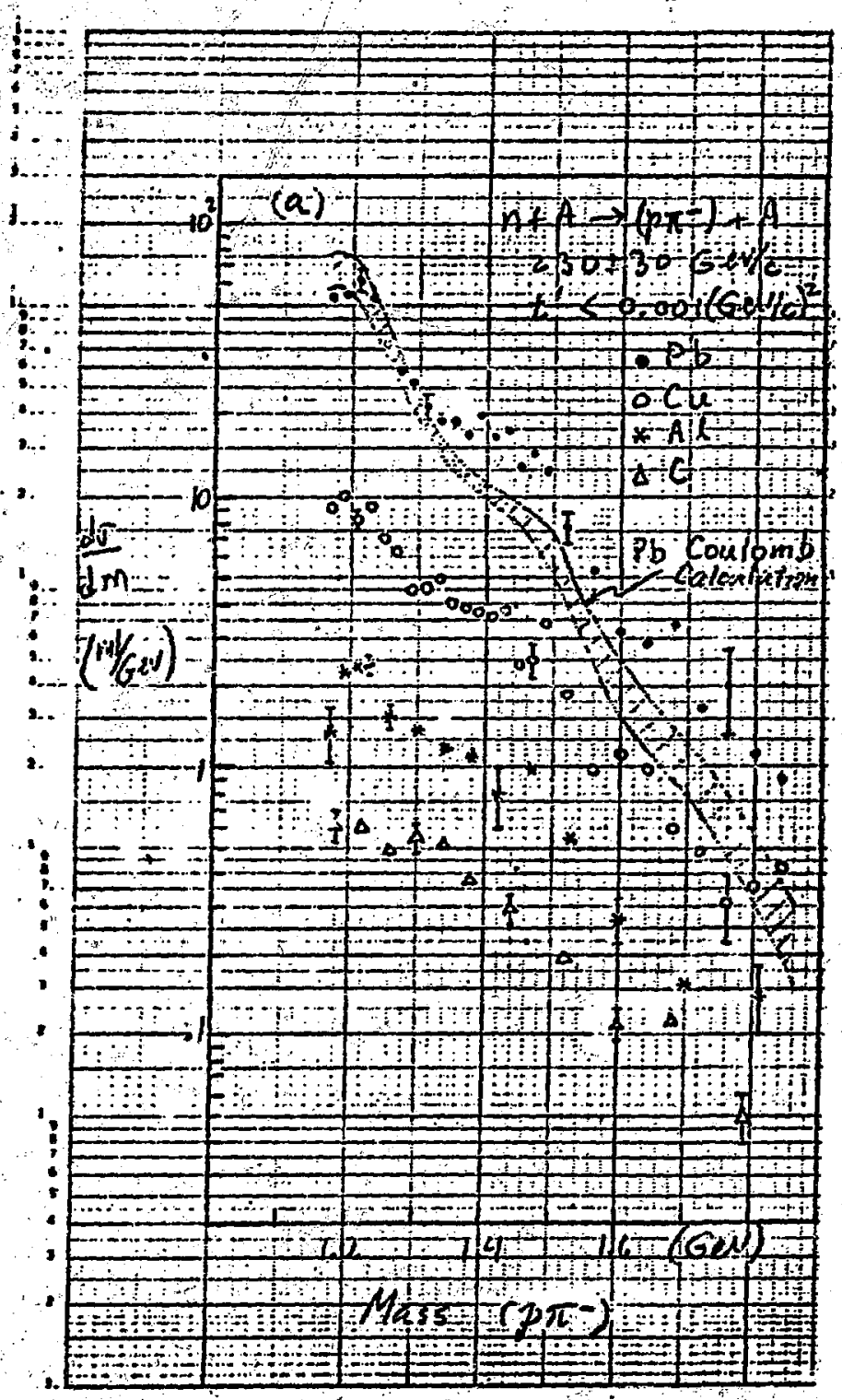

a)

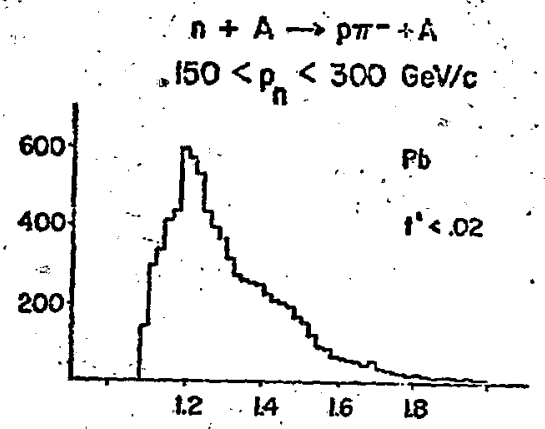

b) $M(p \pi-) G e V / c^{2}$

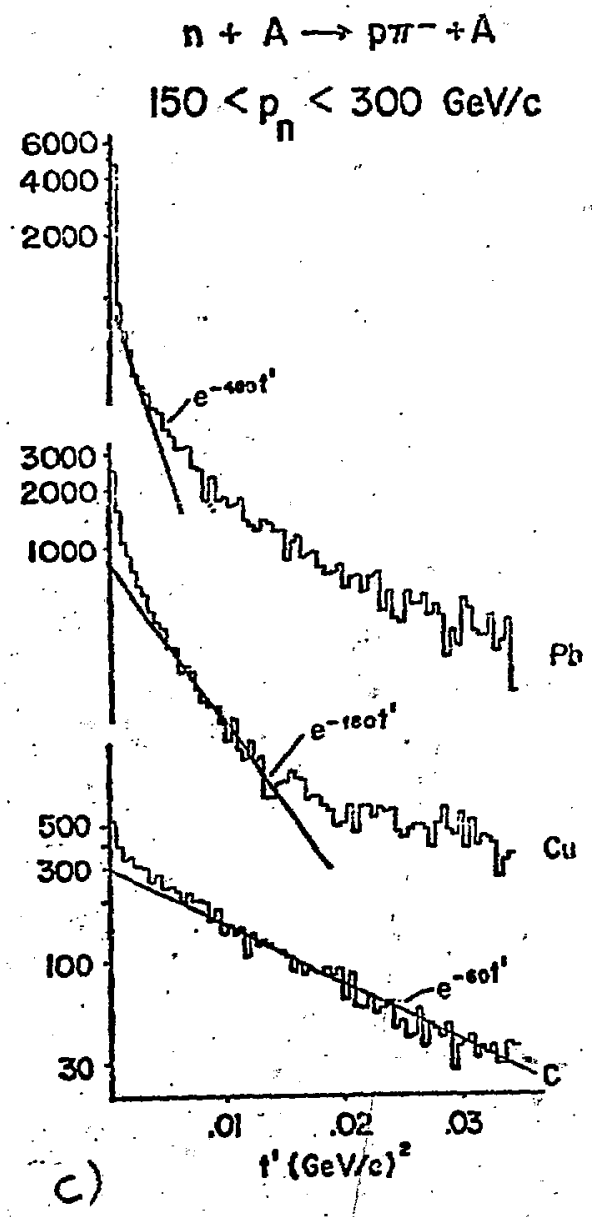

FIGURE 3 : 